\title{
Regulation of Fructose Transport During Growth of Aspergillus nidulans
}

\author{
By MYRA BERMAN KURTZ \\ Waksman Institute of Microbiology, Rutgers - The State University, P.O. Box 759, \\ Piscataway, New Jersey 08854, U.S.A.
}

(Received 17 October 1979; revised 29 December 1979)

\begin{abstract}
Fructose transport by exponentially growing cultures of Aspergillus nidulans declines with culture age. The mechanism of age-dependent decline of transport capacity during exponential growth in liquid culture was studied in a mutant lacking fructokinase. The reduced ability of late cultures to transport fructose was not due to energy limitation as shown by measurements of intracellular ATP concentrations, nor was there a significant change in the apparent $K_{\mathrm{m}}$ of early cultures compared with late cultures. Fructose transport was repressed by growth in glucose and derepressed by starvation. On the addition of glucose to derepressed cultures, fructose transport activity decayed. Comparisons of early and late cultures with respect to synthesis and degradation of the fructose transport system showed that the synthesis of a limiting component of the transport system was reduced in late cultures. The significance of these findings with respect to developmental scheduling is discussed.
\end{abstract}

\section{INTRODUCTION}

In liquid-grown cultures of Aspergillus nidulans, carbohydrate transport (defined as the rate of accumulation of substrate per mg dry weight of mycelium) varies with culture age (Kurtz \& Champe, 1979). Soon after conidial inoculation, the rate of glucose uptake increases, reaches a maximum at about $11 \mathrm{~h}$, and subsequently declines exponentially (Kurtz \& Champe, 1979). Neither starvation nor medium replacement alter the glucose uptake rate, indicating that mycelium of a given age has a characteristic and endogenously controlled transport capacity.

The changing transport capacity has been implicated in the process of developmental timing in $A$. nidulans (Kurtz \& Champe, 1979). Mutants selected for altered developmental scheduling of conidial differentiation lose transport capacity with culture age at different rates from the wild-type (Kurtz \& Champe, 1979). In the slime mould Dictyostelium discoideum, membrane components change during development and, more importantly, these changes affect the developmental programme both in timing and sequence (Siu et al., 1977). In higher eukaryotes, such as early chick embryos, membrane transport and permeability also change with the developmental stage (Kutchai et al., 1977; Grove \& Stockdale, 1978). If the level of transport capacity is a manifestation of an internally controlled timing device for differentiation, it is important to our understanding of development to elucidate the mechanisms regulating transport during growth.

Three general mechanisms for the modulation of transport during growth can be envisaged. Regulation could occur by synthesis and/or degradation of a limiting component of the transport system, such as the carrier protein or the energy supply. Secondly, the activity rather than the synthesis of the transport system could be controlled in a variety of ways. Lastly, new components of the transport system with reduced affinity for the substrate may replace the old ones as the culture ages. 
This communication reports detailed studies of the regulation of fructose uptake at different stages of growth. The fructose uptake system was chosen for study because the availability of a mutant strain lacking fructokinase allows measurement of uptake without the complication of subsequent metabolism (Roberts, 1963). With such a strain, one can assay uptake by the accumulation of a non-toxic substrate (fructose) which is transported by a system quite specific for this sugar (Mark \& Romano, 1971). The results of these experiments suggest that the synthesis of the fructose carrier is regulated differently in early and late cultures.

\section{METHODS}

Strains. Aspergillus nidulans wild-type (Fungal Genetic Stock Collection no. 4) was kindly provided by David Axelrod. Strain 276, a strain lacking fructokinase activity (Mark \& Romano, 1971; Roberts, 1963), was obtained from the Fungal Genetic Stock Collection, Humboldt State University Foundation, Arcata, Calif., U.S.A. In addition to the fructokinase marker, fr $A$, strain 276 carries the mutations $y A$, pyro $A$ and pabaB.

Mycelial growth in liquid culture. The methods used for culture in liquid medium were as previously described (Kurtz \& Champe, 1979). Conidia were inoculated at a final concentration of $10^{5} \mathrm{ml}^{-1}$ into nitrate-less minimal medium (Cove, 1966) as modified by Gealt \& Axelrod (1974), with glucose $(1 \%$, w/v) as carbon source and $0.01 \mathrm{M}$-glutamate as nitrogen source. To meet the vitamin requirements of strain 276 , pyridoxine and $p$-aminobenzoic acid were added to the minimal medium at 50 and $100 \mu \mathrm{g} \mathrm{l}^{-1}$, respectively.

Uptake measurements. Mycelial pellets were harvested, washed and starved for $30 \mathrm{~min}$ for the standard assay as detailed previously (Kurtz \& Champe, 1979). The concentration of fructose used in the standard assay was $1 \mathrm{~mm}$. Uptake rate was measured as nmol fructose accumulated (mg dry wt mycelium) ${ }^{-1} \mathrm{~min}^{-1}$. Modifications of the uptake conditions are noted in the figure legends.

$K_{\mathrm{m}}$ measurements. The apparent $K_{\mathrm{m}}$ for fructose uptake was measured in mycelium prepared as for the standard assay. Assays were initiated by adding $2 \mathrm{ml}$ mycelial suspension to $25 \mathrm{ml}$ pre-warmed phosphate buffer containing radioactively labelled fructose at various concentrations. After 15 min incubation at $37^{\circ} \mathrm{C}$, the reaction was stopped by immediately filtering the mycelium on to membrane filters (Millipore, $0.45 \mu \mathrm{m}$ pore size) and washing with $15 \mathrm{ml}$ water. Dried filters were assayed for ${ }^{14} \mathrm{C}$ in a Nuclear-Chicago gas flow counter. All measurements were performed in duplicate for each substrate concentration and repeated several times with different cultures.

Measurement of intracellular water. Mycelium was prepared as for the standard uptake assay and then filtered on to preweighed Millipore HA filters. To determine total water, the resulting pad was immediately weighed and then dried to constant weight. Parallel samples were mixed with $\left[{ }^{14} \mathrm{C}\right]$ carboxyl-inulin (a nonpenetrating substrate), filtered, dried and counted to determine extracellular water trapped in the mycelial pads. The calculated difference between total water and extracellular water was taken as the intracellular water. The intracellular water varied from one culture flask to another, but was within the range of $2 \cdot 3$ to $2.7 \mu \mathrm{l}(\mathrm{mg} \mathrm{dry} \mathrm{wt})^{-1}$. In a typical experiment, the intracellular water of a $12 \mathrm{~h}$ culture $\left[2.5 \mu \mathrm{l}(\mathrm{mg} \mathrm{dry} \mathrm{wt})^{-1}\right]$ was essentially the same as that of a $20 \mathrm{~h}$ culture $\left[2.6 \mu \mathrm{l}\left(\mathrm{mg} \mathrm{dry} \mathrm{wt}^{-1}\right]\right.$. An average value of $2.5 \mu \mathrm{l}(\mathrm{mg}$ dry wt $)^{-1}$ was used in all calculations in this paper. These values are in good agreement with those previously reported for submerged cultures of Neurospora crassa (Scarborough, 1970).

Preparation of cell extracts for ATP measurements. Mycelium was prepared and incubated for $30 \mathrm{~min}$ in the uptake buffer at $37^{\circ} \mathrm{C}$ as in the standard uptake procedure. At the time of harvest, the cells were collected by filtration on to Miracloth (Chicopee Mills) and immediately plunged into petroleum ether/dry ice. Each frozen pellet was lyophilized, weighed and homogenized in $1.5 \mathrm{ml} 6 \%(\mathrm{v} / \mathrm{v}) \mathrm{HClO}_{4}$ at $0{ }^{\circ} \mathrm{C}$. The samples were extracted for 1 to $2 \mathrm{~h} \mathrm{in} \mathrm{HClO}_{4}$ and then diluted to $5.0 \mathrm{ml}$ and centrifuged at $2000 \mathrm{~g}$ for $15 \mathrm{~min}$. A sample $(4.0 \mathrm{ml})$ of the supernatant fluid was neutralized with $1.2 \mathrm{M}-\mathrm{KOH}$ after adding $1.2 \mathrm{ml} 200 \mathrm{~mm}-$ glycylglycine. The precipitate was removed by filtration through a membrane filter (type RA, $1.2 \mu \mathrm{m}$ pore size, Millipore) and washed with saturated $\mathrm{KClO}_{4}$. Samples were kept frozen until assay. Recovery of ATP was monitored by adding ATP to cell samples after grinding.

ATP measurements. ATP was assayed with firefly luciferase according to the method of Kimmich et al. (1975) using an unmodified Intertechnique scintillation detector at ambient temperature and in a coincidence mode. This method uses $\mathrm{Ca}_{3}\left(\mathrm{PO}_{4}\right)_{2}$-activated crude extracts of firefly lanterns to lower background luminescence and an assay $\mathrm{pH}$ of 8.0 to maximize light emission. For each assay, $0.9 \mathrm{ml}$ assay medium $(5 \mathrm{~mm}-$ sodium arsenate, $4 \mathrm{mM}^{-\mathrm{MgSO}_{4}}$ and $20 \mathrm{~mm}$-glycylglycine, $\mathrm{pH} 8.0$ ) at room temperature and $50 \mu \mathrm{l}$ of appropriately diluted unknown sample or ATP standard were mixed. Then $50 \mu \mathrm{l}$ of activated extract was added to initiate the reaction, and the vial was swirled and lowered into the detection chamber. Exactly $20 \mathrm{~s}$ after adding the extract the sample was counted for a 30 s interval. ATP concentrations in the range of 4 to 20 pmol per $50 \mu \mathrm{l}$ sample could be assayed by this method. 


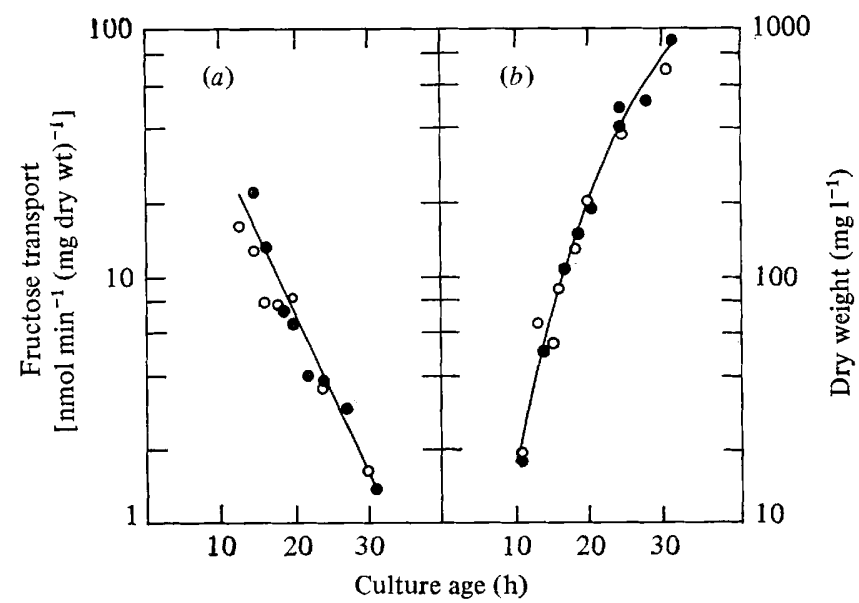

Fig. 1. Transport of fructose $(a)$ and growth rate in liquid culture $(b)$ of wild-type $A$. nidulans strain $4(\bigcirc)$ and fructokinase ${ }^{-}$mutant, strain $276(\bigcirc)$ as a function of culture age.

\section{RESULTS}

\section{Carrier activity, not energy, limits uptake in growing cultures}

To use the mutant strain lacking fructokinase for study of age-dependent changes in fructose transport, it was first necessary to demonstrate that fructose transport declines with culture age in the same way as in the wild-type. Growth and fructose transport as a function of culture age for strain 4 (wild-type) and strain 276 (fructokinase ${ }^{-}$) are shown in Fig. 1. For both strains the rate of change in fructose transport with culture age, as well as growth rate, were the same. It should be noted that the rapid decline in transport ability with age occurred during the period of exponential growth. The remainder of this report describes experiments performed with strain 276 , unless otherwise indicated.

The transport measurements shown in Fig. 1 were made after a 30 min incubation in phosphate buffer to deplete nutrients that might compete with the radioactive substrate. Since this incubation may also have depleted limited energy reserves, the observed difference in transport between early and late cultures might reflect different levels of available energy to drive active transport. To test this possibility, glucose uptake rates with and without incubation were compared in strain 4 . The glucose uptake rate of a $12 \mathrm{~h}$ culture assayed immediately after harvest was $27.2 \mathrm{nmol} \mathrm{min}^{-1}(\mathrm{mg} \text { dry wt })^{-1}$. A $30 \mathrm{~min}$ incubation in phosphate buffer did not substantially alter the rate of uptake $\left[31.1 \mathrm{nmol} \mathrm{min}^{-1}\right.$ (mg dry $w t)^{-1}$. A late culture $(24 \mathrm{~h})$ was no more sensitive to preincubation than the early one:

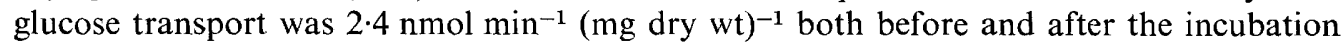
period.

A more direct measure of the cellular energy levels of cultures used for uptake assays may be achieved by determining intracellular ATP concentrations. Age-dependent variation in ATP concentration has been observed in Neurospora cells grown in shaken liquid cultures (Slayman, 1973). However, it was also shown that transfer of growing cells to buffer lacking a nitrogen source stabilizes the internal ATP level near $2.5 \mathrm{~mm}$ [1 mmol (kg cell water $\left.)^{-1}\right]$ independent of cell age. Therefore, ATP determinations were made on extracts prepared from mycelia subjected to the same 30 min starvation in phosphate buffer as used in the uptake assay. The intracellular ATP concentrations of 12,16 and $20 \mathrm{~h}$ cultures were all very similar: $2.73 \pm 0.051,2.75 \pm 0.077$ and $2.65 \pm 0.071 \mathrm{nmol}(\mathrm{kg} \text { cell water })^{-1}$, respectively (average analyses for 3 to 5 cell samples). 


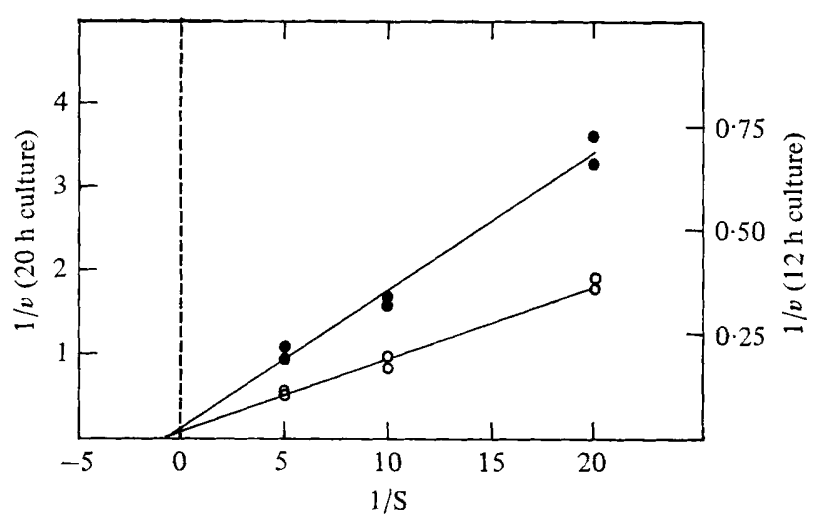

Fig. 2. Apparent $K_{\mathrm{m}}$ for fructose of mycelium from $12 \mathrm{~h}(\mathrm{O})$ or $20 \mathrm{~h}(O)$ cultures [ $\mathrm{v}$ in $\mathrm{nmol} \mathrm{min}^{-1}$ (mg dry wt) ${ }^{-1} ; \mathrm{S}$ (fructose) in $\mathrm{mm}$.

Table 1. Glucose repression of the fructose transport system in A. nidulans strain 4 The growth medium consisted of salts (Gealt \& Axelrod, 1974) with 0.01 M-glutamate as nitrogen source and either $1 \%$ glucose, $1 \%$ fructose, or both, as carbon source.

$\begin{array}{lcc}\text { Carbon source } & \begin{array}{c}\text { Dry wt of culture } \\ \left(\mathrm{mg} \mathrm{l}^{-1}\right)\end{array} & \begin{array}{c}\text { Fructose transport } \\ {[\mathrm{nmol} \mathrm{min})^{-1}} \\ \left(\mathrm{mg} \mathrm{dry} \mathrm{wt}^{-1} \text { ] }\right.\end{array} \\ \text { Glucose } & 230 & 6 \cdot 5 \\ \text { Glucose+Fructose } & 236 & 6 \cdot 5 \\ \text { Fructose } & 215 & 11 \cdot 0\end{array}$

\section{Carrier affinity of growing cultures}

If energy limitation is not responsible for the low transport activity in late cultures, then the low activity may be the result of a physical alteration such that the fructose carrier no longer binds the substrate with the same affinity. Measurements of the apparent $K_{\mathrm{m}}$ for fructose of 12 and $20 \mathrm{~h}$ cultures gave values of 1.0 and $1.25 \mathrm{~mm}$, respectively (Fig. 2). To account for the difference between a 12 and $20 \mathrm{~h}$ culture solely by a change in affinity of the carrier would require that the carrier from a $20 \mathrm{~h}$ culture have a 5 -fold reduction in affinity for fructose. This difference is calculated on the basis of assays with fructose at $1 \mathrm{~mm}$ (Fig. 1), a concentration well below the saturation levels of $5 \mathrm{~mm}$ determined from the data of Fig. 2. The observed difference in $K_{\mathrm{m}}$ between a 12 and $20 \mathrm{~h}$ culture is clearly insufficient to explain the large difference in carrier activity.

\section{Regulation of synthesis of fructose uptake in early and late cultures}

One mechanism for modulating fructose transport during growth could involve regulation of synthesis of a limiting component of the transport system. Previous work has shown (Mark \& Romano, 1971), and the present experiments confirm, that the uptake of fructose is constitutive (Table 1). However, the highest fructose uptake activity was attained in cultures grown on fructose alone, which implies that uptake is repressed by glucose. The results in Figs 3 and 4 show that starvation of a $12 \mathrm{~h}$ glucose-grown culture effects a derepression of the fructose uptake system. The accumulation in a $12 \mathrm{~h}$ sample starved for $30 \mathrm{~min}$ was linear for the entire assay and continued for $60 \mathrm{~min}$ or more (Fig. 3 and results not shown). In contrast, the accumulation of fructose by the freshly harvested mycelium was initially considerably lower for the untreated mycelium than for the preincubated sample, but the rate of accumulation increased continuously with time (Fig. 3).

Figure 4 also shows the effect of cycloheximide on the derepression of fructose transport. This inhibitor of protein synthesis prevented the increase in fructose transport for both 


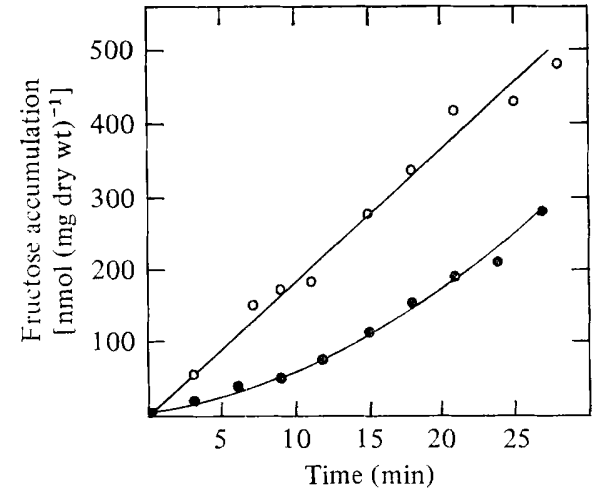

Fig. 3

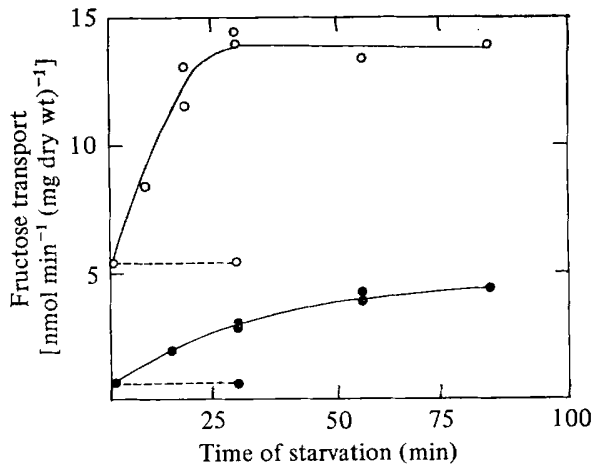

Fig. 4

Fig. 3. Fructose accumulation in a $12 \mathrm{~h}$ culture: starved $(O)$ and not starved $(O)$.

Fig. 4. Effect of starvation time on the extent of derepression of fructose transport. Freshly harvested mycelium was assayed immediately or after various times in phosphate buffer. At 0 time a portion of the culture was removed and cycloheximide $\left(100 \mu \mathrm{g} \mathrm{m}^{-1}\right)$ was added; fructose uptake was assayed $30 \mathrm{~min}$ later. $12 \mathrm{~h}$ culture $(\bigcirc) ; 20 \mathrm{~h}$ culture $\left(\bigcirc_{)}\right),-\ldots$, with cycloheximide; - - , without cycloheximide.

the 20 and $12 \mathrm{~h}$ cultures. These results imply that the increased fructose transport during fasting requires de novo synthesis of the uptake system.

The starvation time required for full derepression is shown in Fig. 4 for both a 12 and $20 \mathrm{~h}$ culture. The early culture was fully derepressed by 20 to $30 \mathrm{~min}$, the standard preincubation time, and no change in rate occurred even after $90 \mathrm{~min}$ in phosphate buffer. Full derepression of later cultures $(20 \mathrm{~h})$ required at least 90 min incubation, but about $70 \%$ of the full activity was synthesized by $30 \mathrm{~min}$. The late culture was able to synthesize the transport system for an hour longer than the early culture, yet the fully derepressed activity of the $20 \mathrm{~h}$ culture was still only $30 \%$ of that of the derepressed $12 \mathrm{~h}$ culture. The percentage of derepression was greater for the $20 \mathrm{~h}$ culture than for the $12 \mathrm{~h}$ culture - a 5 -fold increase for the $20 \mathrm{~h}$ culture, but only a $2 \cdot 5$-fold increase for the $12 \mathrm{~h}$ culture.

From experiments such as that shown in Fig. 4 it is possible to quantify the increasing rate of transport activity during starvation. A comparison of these rates of synthesis of the transport system during starvation for early and late cultures showed that the rate of increase (the initial slopes of the curves shown in Fig. 4) of transport of a young culture is 4 to 5 times greater than a late culture. The ability to respond to the starvation signal is thus not the same in mycelia of different ages.

\section{Degradation of transport activity does not change during growth}

The study has shown that the initial net rate of synthesis of the fructose transport system in response to starvation is greater in early than in late cultures. To see if these results reflect greater degradation of the fructose transport system in the late culture as well as reduced synthetic capacity, the turnover of the transport system in derepressed cultures was measured by inhibiting synthesis with cycloheximide and assaying $30 \mathrm{~min}$ later. The activity was equally stable in both the 12 and $20 \mathrm{~h}$ cultures and did not change under these nongrowing conditions (Table 2).

In Neurospora crassa, inactivation of specific transport systems can be achieved by adding glucose to the medium after derepression (Schneider \& Wiley, 1971). In yeast, a similar loss of transport activity for galactose occurs when glucose is added to the medium (Matern \& Holzer, 1977). This phenomenon, called catabolite inactivation in yeast, has been ascribed to specific proteolytic degradation. An experiment was therefore designed to study the effect of glucose on the stability of the fructose transport system in cultures of different 


\section{Table 2. Stability of derepressed fructose transport}

Mycelium was incubated for $30 \mathrm{~min}$ in buffer to derepress fructose transport (derepressed), then incubated for an additional $30 \mathrm{~min}$ in buffer (starved) or in buffer containing $100 \mu \mathrm{g}$ cycloheximide $\mathrm{ml}^{-1}$ (starved + cycloheximide); fructose transport was assayed for each incubation condition.

\begin{tabular}{|c|c|c|c|}
\hline \multirow[b]{2}{*}{$\begin{array}{l}\text { Culture } \\
\text { age (h) }\end{array}$} & \multicolumn{3}{|c|}{ Fructose transport $\left[\mathrm{nmol} \mathrm{min}^{-1}(\mathrm{mg} \text { dry wt })^{-1}\right]$} \\
\hline & Derepressed & Starved & $\begin{array}{c}\text { Starved }+ \\
\text { cycloheximide }\end{array}$ \\
\hline $\begin{array}{l}12 \\
20\end{array}$ & $\begin{array}{r}14 \cdot 0 \\
3 \cdot 0\end{array}$ & $\begin{array}{r}13.4 \\
3.8\end{array}$ & $\begin{array}{r}14 \cdot 0 \\
3 \cdot 6\end{array}$ \\
\hline
\end{tabular}

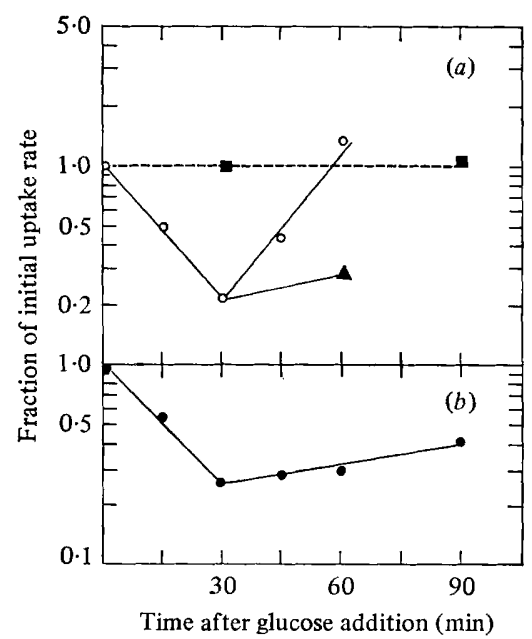

Fig. 5. Effect of glucose and cycloheximide on derepressed fructose uptake activity. Glucose (100 mM final concn) was added at 0 time. (a) 12 h culture $(O)$; control to which no glucose was added (ם); cycloheximide $\left(100 \mu \mathrm{g} \mathrm{ml}^{-1}\right)$ was added to a portion of culture at $30 \mathrm{~min}$ and assayed 30 min later $(\Delta)$. (b) $20 \mathrm{~h}$ culture ( $)$.

ages. Cells grown in glucose were washed and then starved in sugar-free medium for $60 \mathrm{~min}$. Portions were removed and assayed for the initial uptake rate of fructose. At the end of $1 \mathrm{~h}$, glucose was added at a final concentration of $100 \mathrm{~mm}$, and at various times thereafter samples were removed, washed, resuspended in assay buffer and assayed for the initial rate of fructose uptake. Cycloheximide was added to a portion of the culture at $30 \mathrm{~min}$ and assayed $30 \mathrm{~min}$ later. The results (Fig. 5) show that fructose transport declined logarithmically for 30 min after glucose addition to derepressed cultures. The decay rate of the activity was the same in both the 12 and $20 \mathrm{~h}$ cultures, each losing one-half of the original activity in 11 to $13 \mathrm{~min}$. The decay was not altered by the addition of cycloheximide (results not shown). For a $12 \mathrm{~h}$ culture, transport fell to one-tenth of the initial activity by $30 \mathrm{~min}$ from the time of glucose addition. Subsequently the activity increased and by 90 min surpassed the initial level. The $20 \mathrm{~h}$ culture, on the other hand, only partially regained the activity lost on glucose addition, having only one-half of the initial activity after $90 \mathrm{~min}$ incubation. The activity of a control culture, which received no glucose addition, remained the same during the experimental period. Cycloheximide added at $30 \mathrm{~min}$ after glucose addition prevented the appearance of the fructose transport activity. Thus, the reappearance of activity in the glucose-treated cultures was due to de novo synthesis of a fructose transport system and occurred at a faster rate in the early culture than in the late culture. 


\section{DISCUSSION}

The experiments described above show that the age-dependent decline of transport capacity is not due to an energy limitation, but rather to a reduced activity of the transport system. Since the change in the apparent $K_{\mathrm{m}}$ of early cultures as compared to late cultures (Fig. 2) is not sufficient to account for the decline in transport activity with age, it is unlikely that the carrier has been physically modified or replaced by a carrier of lower affinity. The difference in uptake rates could mean either that the early cultures have many more sites for the transport of fructose or that the carriers in the $12 \mathrm{~h}$ cultures transport fructose across the membrane and release it intracellularly at a more rapid rate. An example of loss of carrier sites during differentiation is found in developing chick embryos (Grove \& Stockdale, 1978).

The transport activity in $A$. nidulans appears to be regulated both by the rate and the extent of synthesis of a limiting component of the active transport system, and not by degradation of the transport activity, which is the same in the early and late cultures. The transport activity is not particularly susceptible to inactivation under non-growing conditions; in the absence of protein synthesis full activity is retained for at least $30 \mathrm{~min}$ (Table 2). Glucose stimulates turnover of derepressed activity, but at the same relative rate in the early and late cultures (Fig. 5).

The rate of synthesis of the fructose transport system is greater in a $12 \mathrm{~h}$ culture than in a $20 \mathrm{~h}$ culture under derepressing conditions (glucose starvation) and also in the presence of glucose (glucose-stimulated turnover). It is also clear that the extent of derepression is controlled by age-related factors other than rate of synthesis. If the rate of synthesis of the fructose uptake system were the only difference between a 12 and $20 \mathrm{~h}$ culture, the late culture would eventually reach the same derepressed level as the early culture (Fig. 4), since the proportion of transport activity lost by degradation is the same at each age (Fig. 5).

The fructose transport capacity is regulated in $A$. nidulans both by culture age and glucose repression. The level set by growth in medium containing glucose is disproportionately lower in late cultures than in early ones, as evidenced by the greater relative increase on derepression (Fig. 4). After full derepression by starvation, the activity of the transport system rapidly decays on glucose addition. A similar degradation of transport activity has been demonstrated for Neurospora crassa (Schneider \& Wiley, 1971) and Saccharomyces cerevisiae (Matern \& Holzer, 1977). However, in these fungi the degradation continues for $2 \mathrm{~h}$ without any sign of recovery in contrast to resumption of synthesis observed for fructose transport in $A$. nidulans. Recovery requires protein synthesis and results in activity somewhat higher than the initial fully derepressed levels for an early culture. The means by which glucose repression is overcome in this case is not known. Glucose may induce a non-specific carrier which is turned on only after a low level of uptake is experienced.

Several mechanisms could operate to produce the observed reduction with age in the rate of synthesis of the fructose transport system, including an alteration in the specificity of the protein-synthesizing machinery with age such that some proteins (luxury, inducible enzymes) are not produced at the same rates as more essential ones. Gealt \& Axelrod (1974) suggested this mechanism to explain their observation that, after developmental competence is achieved in $A$. nidulans, the inducibility of protease and nitrate reductase is greatly reduced, whereas the specific activities of constitutive enzymes such as glucose-6-phosphate dehydrogenase and hexokinase do not change with culture age (Gealt \& Axelrod, 1974; Kurtz \& Champe, 1979). The fact that uninduced acetate uptake does not decrease between 12 and $20 \mathrm{~h}$ of culture (M. B. Kurtz, unpublished results) suggests that a changing cell wall content is not responsible for the decline in fructose uptake rate. An alternative model suggests a limitation on the number of sites available for insertions of the carrier in the membrane caused possibly by a changing composition of either lipid or protein components. 
In Neurospora the amount of lipid per g dry weight is constant during germination and growth, but the proportion of phospholipid varies with the age of the culture (Beck \& Greenawalt, 1977). Although the protein components of the membrane of Aspergillus are largely conserved during growth in submerged culture, several membrane proteins disappear (M. B. Kurtz, unpublished results). These proteins may determine some of the physical characteristics of the membrane. Further studies along the lines suggested should permit a choice between the alternative models and a clearer understanding of developmental scheduling in Aspergillus.

I would like to thank Sewell P. Champe for providing laboratory facilities for this work and for stimulating discussions. I am indebted to A. Chang for assistance and to L. Yager, N. Butnick and N. E. L. Hall for helpful suggestions. This investigation was supported in part by Public Health Service grant GM 17020 from the National Institute of General Medical Sciences to S. P. Champe and the Charles and Johanna Busch Memorial Fund.

\section{REFERENCES}

Beck, D. P. \& Greenawalt, J. W. (1977). Composition and synthesis of cellular lipids in Neurospora crassa during cellular differentiation. Journal of Bacteriology 131, 188-193.

Cove, D. J. (1966). The induction and repression of nitrate reductase in the fungus Aspergillus nidulans. Biochimica et biophysica acta 113, 51-56.

Gealt, M. A. \& Axelrod, D. E. (1974). Coordinate regulation of enzyme inducibility and developmental competence in Aspergillus nidulans. Developmental Biology 41, 222-232.

Grove, B. K. \& Stockdale, F. E. (1978). Membrane function in differentiating skeletal muscle cells $I$. Kinetic analysis of amino acid transport. Developmental Biology 66, 142-150.

Kimmich, G. A., Randles, J. \& Brand, J. S. (1975). Assay of picomole amount of ATP, ADP and AMP using the luciferase enzyme system. Analytical Biochemistry 69, 187-206.

Kurtz, M. B. \& Champe, S. P. (1979). Genetic control of transport loss during development of Aspergillus nidulans. Developmental Biology 70, 82-88.

Kutchai, H., King, S. L., Martin, M. \& Daves, E. D. (1977). Glucose uptake by chicken embryo hearts at various stages of development. Developmental Biology 55, 92-102.
Mark, C. G. \& Romano, A. H. (1971), Properties of the hexose transport systems of Aspergillus nidulans. Biochimica et biophysica acta 249, 216-226.

Matern, H. \& Holzer, H. (1977). Catabolite inactivation of the galactose uptake system in yeast. Journal of Biological Chemistry 252, 63996402.

RoBerts, C. F. (1963). The genetic analysis of carbohydrate utilization in Aspergillus nidulans. Journal of General Microbiology 31, 45-58.

Scarborough, G. A. (1970). Sugar transport in Neurospora crassa. Journal of Biological Chemistry 245, 1694-1698.

SchNeIDER, R. P. \& Wiley, W. R. (1971). Regulation of sugar transport in Neurospora crassa. Journal of Bacteriology 106, 487-492.

Siv, C. H., Learner, R. A. \& Loomis, W. F. (1977). Rapid accumulation and disappearance of plasma membrane proteins during development of wildtype and mutant strains of Dictyostelium discoideum. Journal of Molecular Biology 116, 469479.

Slayman, C. L. (1973). Adenine nucleotide levels in Neurospora as influenced by conditions of growth and by metabolic inhibitors. Journal of Bacteriology 114, 752-766. 